\title{
START-UP AND SAFEGUARDING OF AN INDUSTRIAL ADIABATIC TUBULAR REACTOR
}

\author{
J. W. VERWIJS, $†$ H. VAN DEN BERG† and $K$. R. WESTERTERP †* \\ $\nmid$ Process Development \& Control Department, Dow Benelux NV, 4530 AA Terneuzen, The Netherlands \\ and $\ddagger$ Chemical Reaction Engineering Laboratories, Department of Chemical Engineering, Twente \\ University, 7500 AE Enschede, The Netherlands
}

(Received 11 May 1994; accepted for publication 27 Septemher 1994)

\begin{abstract}
The safeguarding methodology currently used in the chemical industry is based on controlling the instantaneous values of the process state variables within a certain operating window, the process being brought to shut-down when the operating constraints are exceeded. It is concluded from an analysis of runaways which occurred in industrial reactors that this safeguarding methodology does not necessarily prevent reactor systems suffering from a runaway because (a) excessive amounts of unreacted chemicals can still accumulate in the process, and (b) no means are provided to the operating personnel of identifying such hazardous process deviations during dynamic operations. A model-based start-up and safeguarding procedure is developed for an industrial adiabatic tubular reactor to improve process safety during start-up operations. The trajectories of the manipulated variables are optimized by minimizing the breakthrough of one of the main reactants in the reactor effluent. A maximum reactor temperature constraint is also taken into account. It is concluded that a proper control of the initial reactor temperature profile is critical for a safe reactor start-up while the impact of the other manipulated variables is relatively small in comparison to the effect of the initial reactor temperature profile.
\end{abstract}

\section{INTRODUCTION}

The safety record of the chemical industry is generally good in comparison with other industries (Lees, 1991). Nevertheless incidents involving hazardous chemical reactions continue to happen (Hancock, 1992) and the consequences of such incidents may be dramatic due to the resulting loss of human life and/or the scale of property damage (Garrison, 1988; Mahoney, 1990, 1993). Therefore, many studies have been carried out on reactive chemicals hazard assessment resulting in guidelines for batch and semi-batch reactor design, control and operation [see e.g. the IChemE guideline edited by Barton and Rogers (1993)].

The research for continuously-operated tubular reactors has almost been limited to thermal runaway prevention in (cooled) fixed-bed reactors. In this domain the works of Hosten and Froment (1986), Morbidelli and Varma (1989), Bauman et al. (1990) and Raghaven (1992) can be mentioned, without being exhaustive. It resulted in design criteria to detect operating regions of parametrically sensitive behavior. In parametrically sensitive operating regions, relatively small changes in reactor inlet conditions or other physical parameters can lead to large excursions in the operating conditions (= runaway behavior).

Infortunately the research on thermal runaway phenomena in (cooled) fixed-bed tubular reactors is

\footnotetext{
To whom correspondence should be addressed
}

limited to model calculations only. A comparison of the theoretical results with experimental data from industrial reactors is not available because too few incidents have been thoroughly documented in the open literature for research purposes.

The dynamic behavior of an industrial continuously-operated adiabatic tubular reactor is described in this article. Experimental data of a reactor start-up and of a thermal runaway are used to demonstrate some problems which may be experienced during such events. These examples will also be used to demonstrate that the safeguarding methodology commonly used in the chemical industry does not necessarily prevent an exothermic chemical reactor from a runaway.

\section{RUNAWAY BEHAVIOR CLASSIFICATION}

In this study a runaway is defined as a large excursion from the expected operating conditions of a reactor, like temperature, pressure or material composition. This definition is much broader than that of the thermal runaway phenomena usually referred to. The definition is extended because upsets in continuously-operated reactor systems can have a serious safety impact on other units in the process, and vice versa. For example, many continuously-operated industrial reactors require a complete conversion of one of the main reactants at the reactor exit to avoid the formation of mixtures of reactive chemicals in process equipment which has not been designed for exothermal chemical reaction conditions. Hence, 
(large) excursions from the expected reactor effluent composition are not allowed in such cases.

From this process operation point of view, three categories of runaway behavior can be defined for continuously-operated (tubular) reactor systems.

\begin{abstract}
Runaway from (close to) normal steady-state conditions The major risk for a runaway from (close to) normal steady-state conditions originates from equipment failures, control failures or from flow maldistribution effects because a tubular reactor is operated normally in a region which is not sensitive to runaway behavior to maintain the process economics (Westerterp et al., 1984; Westerterp and Ptasinski, 1984; Westerterp and Overtoom, 1985; Westerink and Westerterp, 1988). An industrial example, including some experimental data, is reported by Eigenberger and Wegerle (1982). They observed a thermal runaway reaction in an adiabatic packed-bed reactor in which liquid hydrocarbons were partially hydrogenated. The runaway was caused by obstructions in the packed bed which led to areas with abnormally high fluid residence times where the reaction could proceed up to total methanation.
\end{abstract}

Runaway from expected process trajectories during dynamic operations The dynamic behavior of a process system is a function of the initial process conditions, process disturbances and the trajectories of the manipulated process variables. Hence, a runaway during dynamic process operations, like start-up, shut-down and capacity-rate changes can be initiated by "false" start conditions, insufficient control of the manipulated variables, flow maldistribution effects, equipment failures or control failures.

Naess et al. (1993) reported some experimental data of serious temperature control problems in an ammonia synthesis reactor caused by control loop instabilities when the gas flow to the reactor was reduced by about $50 \%$. The resulting reactor temperature oscillations, with a maximum amplitude of about $160 \mathrm{~K}$, damaged the catalyst activity partially.

Runaway from expected process conditions initiated by upsets in process sections upstream of the reactor Operational problems in other process sections can strongly influence the quality of the reactant and utility feeds to the reactor which may result in temperature control problems, or in reactant or catalyst reactivity changes.

No examples are known to the present authors of published experimental data of this type of runaway incidents in industrial continuously-operated tubular reactors. However, to stress the importance of being aware of this kind of process hazards, an incident which was experienced recently in a hydrogenation reactor (Shewbart, 1993) should be mentioned. This hydrogenation reactor suffered a thermal runaway while going through a catalyst regeneration sequence. Hydrogen gas was flowing through the catalyst bed for catalyst reduction. At the same time the hydrogen plant had an upset and hydrocarbons entered the hydrogen feed stream into this reactor. The result was an excessive temperature rise in the catalyst bed.

\section{INDUSTRIAL PROCESS}

The adiabatic reactor is shown schematically in Fig. 1, together with its upstream and downstream process sections. The process is shown in a simplified non-heat-integrated form in order not to reveal confidential information. In this reactor the products, denoted by $C$, are formed by an exothermal liquid phase reaction between the reactants $A$ and $B$ :

$$
\mathrm{A}+\mathrm{B} \rightarrow \mathrm{C}
$$

Reactant A is fed from storage into a buffer tank, which also receives recycled material from a recovery unit. Reactant $A$ is fed from the buffer tank into the reactor together with reactant $B$, which is fed from storage. The adiabatic reactor system consists of a feed mixer, a preheater and a series of seven vessels with baffles, of which only one vessel is shown in Fig. 1 for the sake of simplicity. A detailed description of this plant reactor is presented by Verwijs et al. (1992).

The performance of this reactor indicates that it can be characterized as a tubular reactor. Reactant $A$ is fed in excess because reactant $B$ should be totally converted at the reactor exit to avoid the formation of reactive chemical mixtures elsewhere in the process. The excess amount of reactant $A$ is recovered from the reactor effluent and recirculated into the intermediate storage tank. The entire reactant $A$ recovery section is represented by a single distillation column in Fig. 1. The crude product is discharged from the recovery unit into the product refining section.

The reactant $B$ flow rate is controlled by a valve in the $B$ feed line at a setpoint defined by a production capacity target. The reactant A flow rate is controlled in ratio with the reactant $B$ flow by a valve in the reactor effluent line. ' $T$ he reactor effluent flow rate is recorded only. The reactor inlet temperature is controlled by a valve in the steam supply line to the preheater at a setpoint which corresponds with the total feed rate into the reactor. The temperature along the reactor is recorded by nine thermoelements, which are located at several positions between the reactor inlet $(z=0)$ and outlet $(z=1)$. The locations of the control valves, the flow devices (F) and some thermoelements (T) are also indicated in Fig. 1.

\subsection{Conventional safeguarding methodology}

The plant reactor is safeguarded at normal operating conditions with respect to mass flow by monitoring:

- The actual reactant $B$ flow rate into the reactor versus the flow setpoint.

- The actual flow-ratio of reactant $A$ and $B$ into the reactor versus the flow-ratio setpoint.

- The mass balance over the entire reactor by comparing the mass-flow of both reactant feed streams with the reactor effluent stream. 


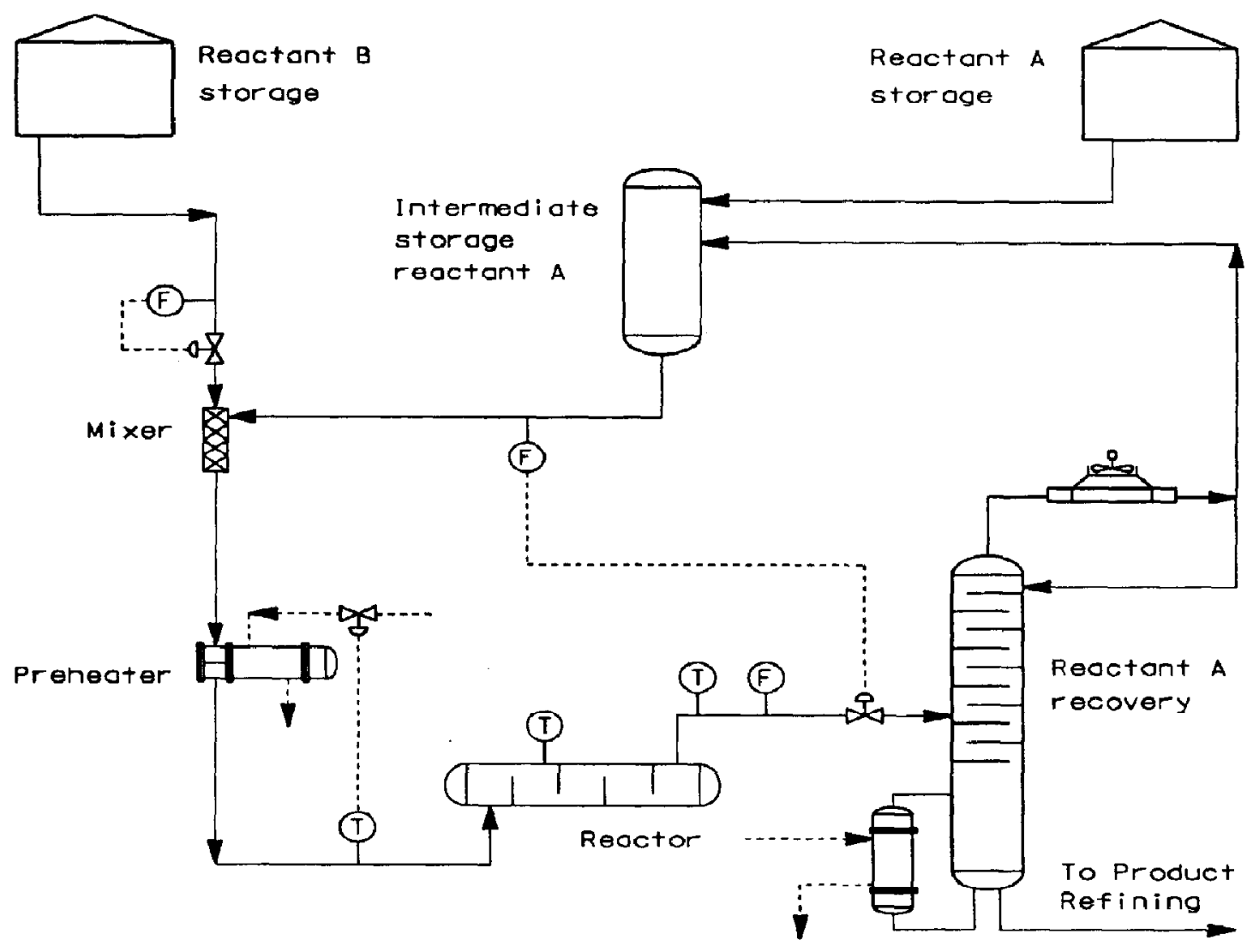

Fig. 1. Process scheme.

Additionally, the adiabatic reactor is safeguarded with respect to temperature by monitoring:

- The reactor inlet temperature versus the inlet temperature setpoint $\left(\Delta \theta_{\text {in }}\right)$.

- The adiabatic temperature rise over the entire reactor versus a target value $\left(\Delta \theta_{\mathrm{ad}}\right)$ which depends on the flow-ratio of reactant $A$ and $B$ in the reactor feed.

- The temperature difference over the tail-end section of the reactor versus a maximum allowable value $\left(\Delta \theta_{\text {iso }}\right)$ which corresponds with the total reactant $B$ conversion requirement and takes temperature measurement inaccuracies into account.

- The reactor temperatures at all thermoelement locations versus a maximum and a minimum allowable reactor temperature constraint $\left(\theta_{\max }\right.$ and $\theta_{\min }$ ).

A typical, steady-state temperature profile over the entire reactor length $z$ is shown in Fig. 2. The dimensionless temperature $\theta$ rises from a value of about 0.98 at the inlet up to about 1.30 at the exit. Reactant $B$ has been converted totally at about $z=0.8$, which is indicated by the flat temperature profile between $z=0.8$ and $z=1.0$. The above-mentioned temperature safeguarding items are indicated as well in Fig. 2. To the authors' best knowledge, such a safeguarding strategy is quite common in the chemical industry. Of course, different measures from those mentioned above may be used for a particular reactor system, but the key issue of controlling the instantaneous values of the process state variables within a certain operating window will be the same.

\section{EXAMPLE 1: RUNAWAY FROM STEADY-STATE CONDITIONS}

This plant reactor suffered a thermal runaway (Verwijs et al., 1994a, 1994b). Experimental data had been collected of the reactant $A$ feed, the reactant $B$ feed and the reactor effluent flow rate. These flow data are presented as a function of the dimensionless time $\sigma$ in Fig. 3. The data are expressed as a percentage of the range of the individual flowmeters in order to not reveal confidential information. The reactant $A$ flow device failed at time $\sigma \approx 0.47$ resulting in an erroneous flow measurement. This flowmeter indicated that the flow rate started rising slowly to the maximum range of the device. The ratio controller 


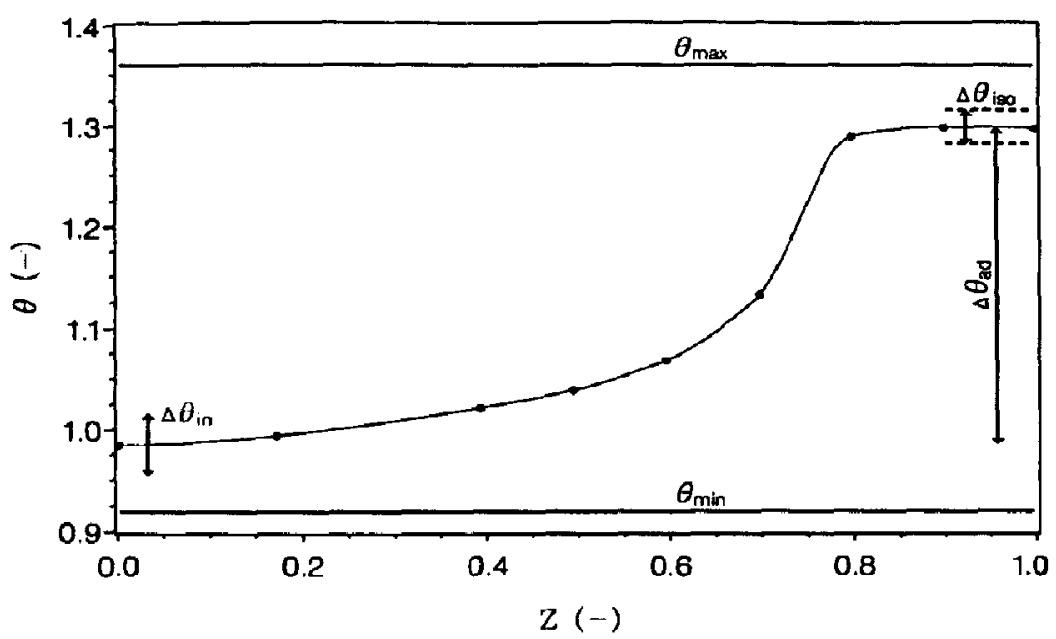

Fig. 2. Observed steady-state temperature profile over the reactor length $z$.

responded to this increasing flow signal by closing the reactant $A$ flow control valve in the reactor effluent line, which can be seen from the total reactor effluent flow data in Fig. 3. During this event the reactant B flow rate was maintained at its setpoint value. As a result, the reactants were fed in a wrong ratio into the reactor. The process control computer stopped the reactant $B$ feed to the reactor at time $\sigma \approx 1.3$ due to a low reactor inlet temperature.

The responses of the nine thermoelements along the entire reactor length are shown in Fig. 4. The time at which the reactant $A$ flow device failed, and at which the reactant B pump was stopped, are marked with a vertical solid line in Figs 3 and 4 . The thermoelements at $z=0.39, z=0.49, \quad z=0.60$ and $z=0.70$ responded very quickly to the flow device failure. The reactor temperatures at these locations increased hecause of the decreasing total flow rate through the system and the reactant ratio change. Note that it took a time interval $\Delta \sigma \approx 0.8$ before the reactor inlet temperature exceeded the minimum reactor inlet temperature constraint and the reactant $B$ feed pump was stopped.

The thermal runaway was initiated by a failing flow device. The failure was observed by the process control computer which compares the mass-flow of both reactant feed streams with the reactor effluent stream. The process control computer did not shut down the reactor system automatically but only gave an alarm. At that point in time the operators were not aware of the upcoming runaway because all reactor temperatures were still in the normal operating range, and they restarted the reactant $B$ feed pump to return to the normal operating conditions [see Verwijs $e t$ al. (1994a) for details]. Later on, when the operators noted the imminent runaway, they responded very proficiently to the emergency situation. They kept the reactant $A$ pump running and closed the control valve in the reactor effluent line to pressurize the system. In this way evaporation of reactant $B$ from

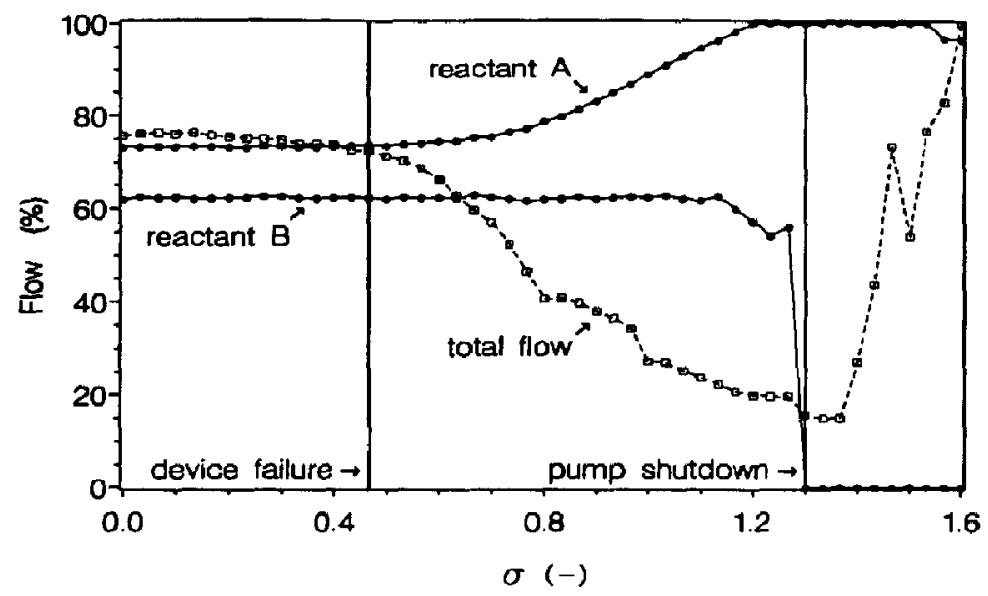

Fig. 3. Observed reactant $A$, reactant $B$ and total flow as a function of time during the temperature runaway. 


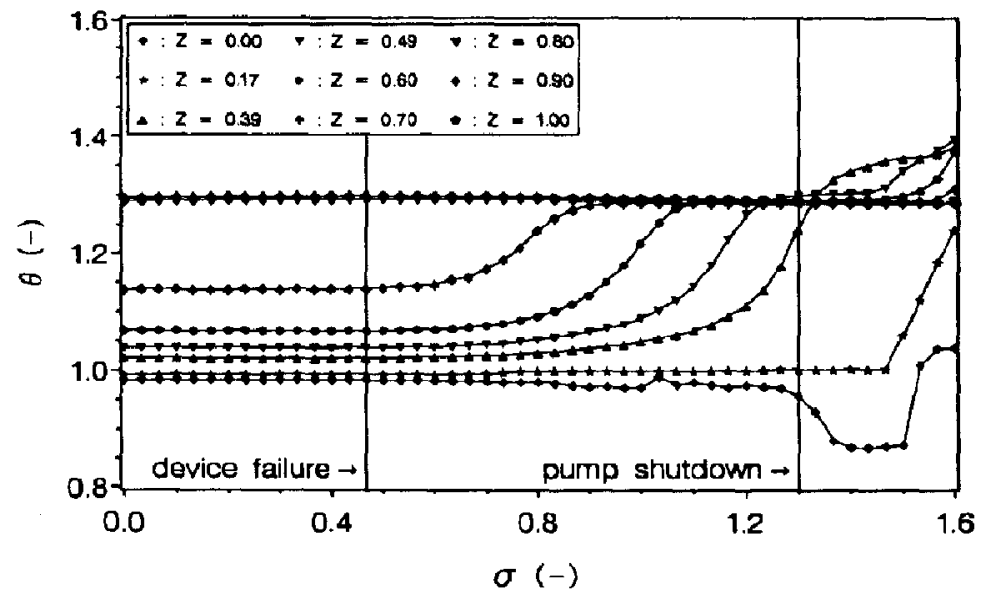

Fig. 4. Observed reactor temperature as a function of time at various reactor locations $z$ during the temperature runaway.

the liquid phase was prevented and almost no reactant B was released into the downstream process section.

\subsection{Learning experiences of Example I}

When assessing the hazards associated with exothermic chemical reactions it is essential to determine how much reactant accumulates in the process. Excessive amounts of accumulated reactants will cause high reaction rates and consequently high heat production rates, or in this particular case an excessive adiabatic temperature rise. Correct flow control and mass balance control is only one aspect of reactant accumulation prevention. It must be kept in mind that instruments and control devices will fail eventually. Therefore the question of redundancy for control system components must be considered thoroughly; this problem as such is beyond the scope of this study. Some guidelines for selecting the appropriate level of redundancy are given by Englund and Grinwis (1992).

As mentioned above, a process is usually safeguarded by controlling the instantaneous values of the process state variables within certain operating windows, and corrective actions are taken when the value of a process state variable exceeds a certain constraint. Usually these operations are executed automatically by hard-wired emergency shutdown systems when the process safety constraints are exceeded.

As can be seen from Figs 3 and 4 , the mass balance control of the reactor failed at $\sigma \approx 0.47$, but the system was able to maintain the reactor temperatures within the selected temperature constraints until $\sigma \approx 1.3$. During this period reactant $B$ accumulated in the system. As a result the temperature rise could no longer be stopped by the operating personnel when the reactor temperature exceeded the maximum temperature constraint due to the heat of reaction. This aspect is the key difference with process units in which no exothermic reaction is carried out. For such units the heat input into the system can be stopped as soon as a maximum temperature constraint is exceeded and the process temperature will return to normal operating conditions. In the reactor incident case studied, the possible maximum temperature rise is determined by the amount of reactants accumulated in the system. Hence, when the reactor temperature exceeded the maximum allowable temperature constraint, and the heat input and/or the reactant feeds were stopped, the system did not return to the normal operating conditions and the thermal runaway proceeded.

It can be concluded from this thermal runaway example that controlling the instantaneous values of the process state variables within certain operating windows is not sufficient to prevent the system from a runaway, because if one line of defence fails (in this example mass balance control) the other line of defence (temperature control) does not necessarily take over in time.

This thermal runaway would not have occurred if the flow meter had not failed. Hence, in industrial practice people focus on installing the appropriate level of redundancy for process control system components (Englung and Grinwis, 1992). In this way, it becomes much more difficult to pass the lines of defence based on mass or heat balance (temperature) control. Of course, a properly installed and a sufficiently redundant safeguarding system will reduce the risks for runaways to a large extent at normal steady-state operating conditions. So one could argue that the safeguarding strategy seems to be sufficient when the mass balance control system is in good order.

But this is not necessarily the case, as can be explained by comparing the above-described safeguarding strategy with the runaway modes and causes defined in the previous section. The thermal runaway example in the hydrogen reactor during the catalyst regeneration sequence (Shewbart, 1993) could not have been prevented by proper mass balance control. In this case the mass balance over the reactor would have been measured to be in good 
order but the reactivity of the reactants changed during the catalyst reduction step. This reactant reactivity change can only be detected by controlling the energy balance and/or the temperature profile over the entire reactor in conjunction with the reactor mass balance. Also, the thermal runaway due to flow maldistribution effects (Eigenberger and Wegerle, 1982) could not have been prevented by proper mass balance control only. In such circumstances, an imminent runaway can be delected only by controlling the temperature profile over the entire reactor in conjunction with the reactor mass balance.

The key issue of the safeguarding strategy described is that the mass and heat balances over the reactor are uncoupled by controlling the reactant flows and some reactor temperatures as such within certain operating windows. Due to this aspect no online information is available about the extent of the reaction taking place, and reactants can accumulate relatively easily in the system, especially at abnormal process conditions. Hence, to avoid an unexpected reactant accumulation in the system the temperature profile along the entire reactor length has to be included in the reactor safeguarding strategy. Also, the spatially distributed nature of the process conditions over the entire reactor length complicates considerably the implementation of a control and safeguarding system based on the reactor temperature profile, because the temperature profile depends on the reactor operating conditions, such as total flow, reactant ratio and reactor inlet temperature. Therefore, a model-based process control and safeguarding system is required to control the reactor mass balance in conjunction with the reactor heat balance. In this way, online information can be obtained from the model about the extent of the reaction, even at abnormal process conditions.

\section{EXAMPLE 2: RUNAWAY DURING START-UP OPERATIONS}

In reality the reactant $A$ recovery system is much more complex than shown in Fig. 1 and highly heatintegrated. Also, no auxiliary systems were available to operate the recovery system before the reactor was started up. Hence, the reactor and the reactant $A$ recovery unit were started up simultaneously.

The entire process system was prepared for start-up by filling the system via the nomal how route with a mixture of reactant $A$ and product $C$. The material is heated up in the reactor preheater simultaneously. These operations were executed before the actual start-up; see also Verwijs et al. (1992, 1994a, 1994b). The reactor start-up procedure followed was to feed reactant $B$ into the reactor as soon as the reactant $A$ flow rate and the reactor inlet temperature reached the target values used at normal steady-state conditions, in order to minimize the amount of offgrade material produced during start-up operations.

Experimental data on the reactant $A$ and $B$ flows, and the reactor pressure, are shown in Fig. 5 as a function of the dimensionless time $\sigma$. The data are expressed as a percentage of the range of the particular pressure and flow measurement devices. The time $\sigma$ is scaled by taking the time origin $(\sigma=0)$ just before reactant $B$ is added into the system (Verwijs et al., 1992). As a result of this time origin choice, time $\sigma$ appears to be negative in Figs 5 and 6. The dimensionless temperature $\theta$ over the entire reactor is shown in Fig. 6. In this figure, the lines parallel to the $\sigma$-axis represent the response of the individual thermoelements at the dimensionless location $z$, and the lines parallel to the $z$-axis connect the data at the same moment. The $z$-axis is scaled from $z=0$ at the reactor inlet to $z=1$ at the exit. Note that in Fig. 6, the $\sigma$ axis is drawn from the right side to the left.

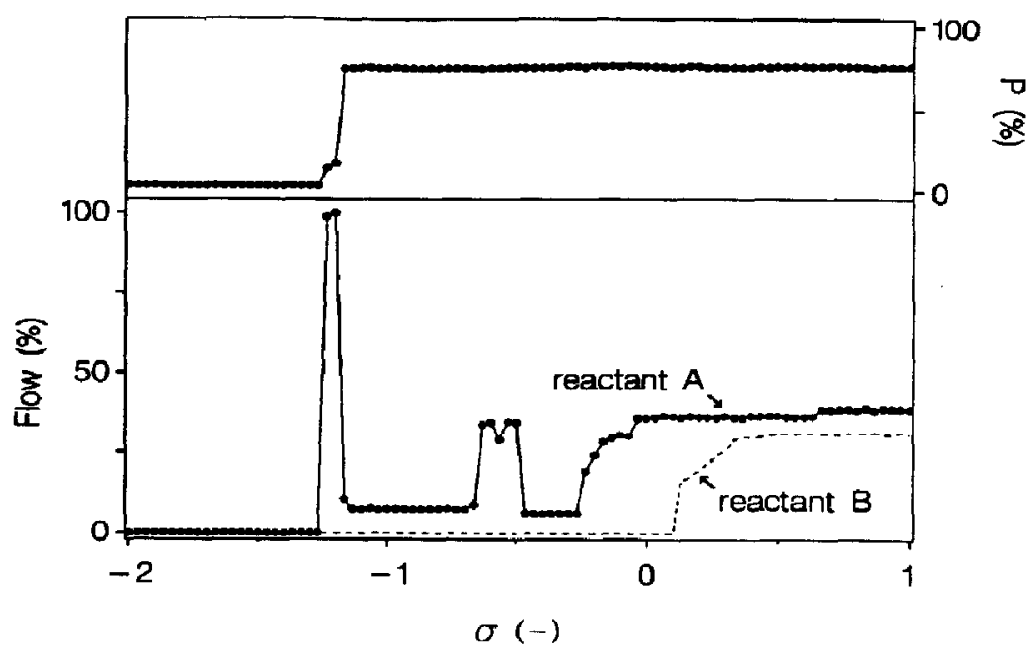

Fig. 5. Observed reactor pressure and flow of reactants A and B as a function of time. 


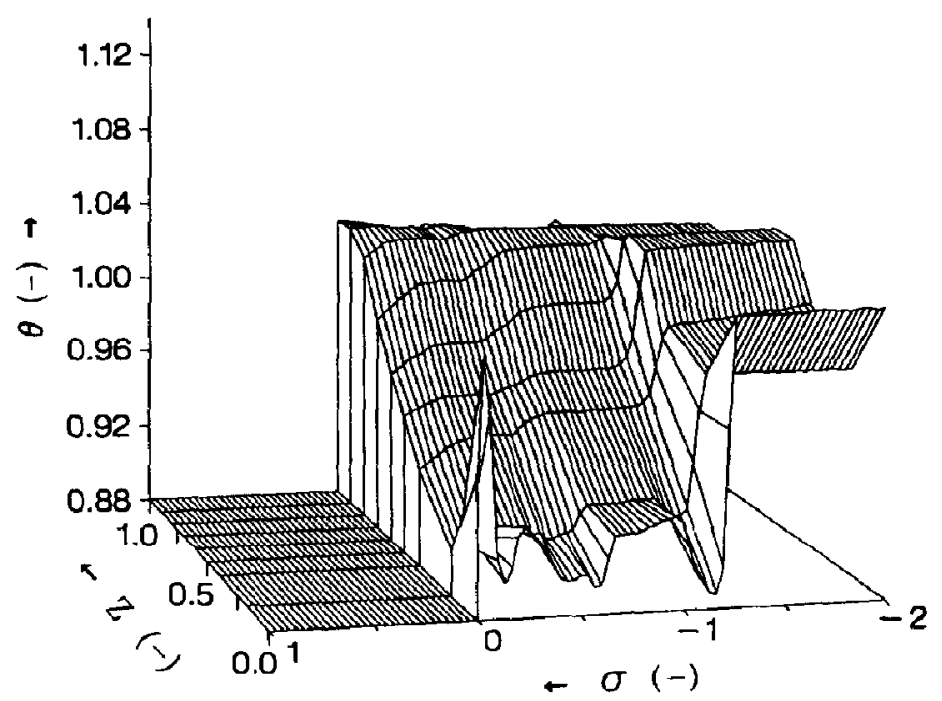

Fig. 6. Observed reactor temperature versus reactor location and time.

The operations executed to fill the system with process material are completed before the period of time of which the data are presented. The temperature profile $\theta$ over the entire reactor length $z$ at time $\sigma=-2.0$ in Fig. 6 resulted from these operations. During the period $\sigma=-2.0$ until $\sigma=-1.27$, the reactor is waiting for the next operational action. At $\sigma=-1.27$, virginal reactant $\mathrm{A}$ is fed into the reactor; see Fig. 5. The system was not completely filled up with liquid at that time, and the reactant $A$ flow was raised initially to maximum capacity until the reactor pressure reached the normal operating value. During the period $\sigma=-1.27$ until $\sigma=-0.14$ relatively cold material entered the reactor. At $\sigma=-0.14$ steam is fed into the feed preheater to control the reactor inlet temperature. All these process manipulations resulted in the initial temperature profile $\theta$ at $\sigma=0$ shown in Fig. 6 .
The reactor behavior from $\sigma=0$ and onwards is shown in Figs 7 and 8. At $\sigma=0.10$, the feed-pump of reactant $B$ is started up (see Fig. 7). During the period $\sigma=1.47$ until $\sigma=1.70$, no reactant $B$ is fed into the system due to a pump failure. The reactant $B$ feed-pump is restarted at $\sigma=1.70$. At $\sigma=3.00$, the reactor feed is ramped up to a minimum plant capacity which is reached at $\sigma=5.50$. The resulting temperature profile $\theta$ over the entire reactor from time $\sigma=0$ and onwards is shown in Fig. 8.

\subsection{Learning experiences of Example 2}

As mentioned before, a breakthrough of reactant $B$ in the reactor effluent is not allowed for process safety reasons. However, it was observed that significant amounts of reactant $B$ could end up in the reactor effluent by applying the start-up methodology described above. Therefore, a modeling study was

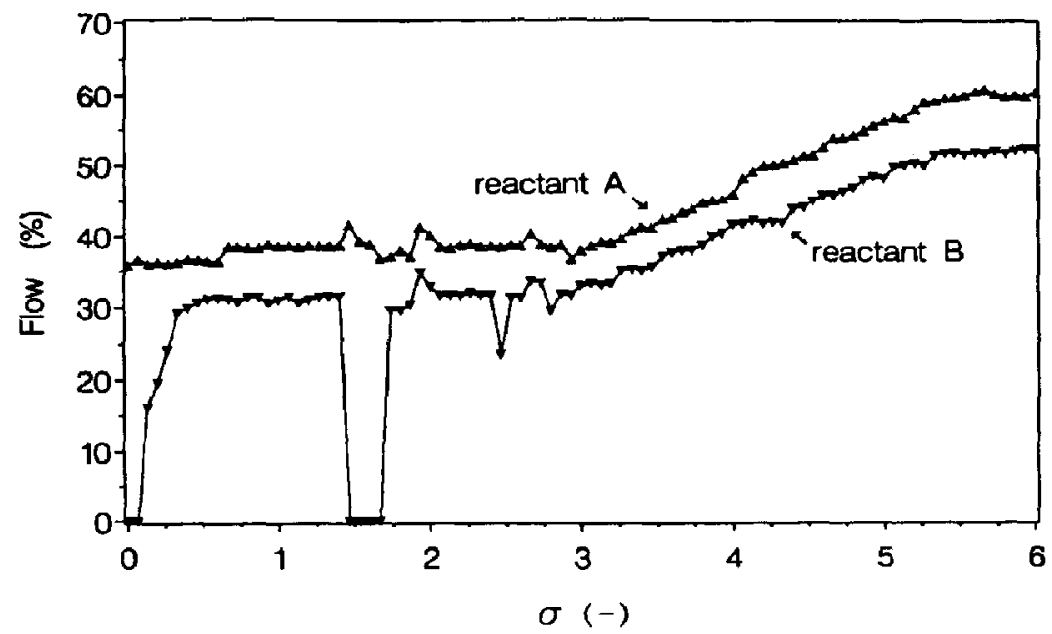

Fig. 7. Observed flow of reactants A and B as a function of time. 


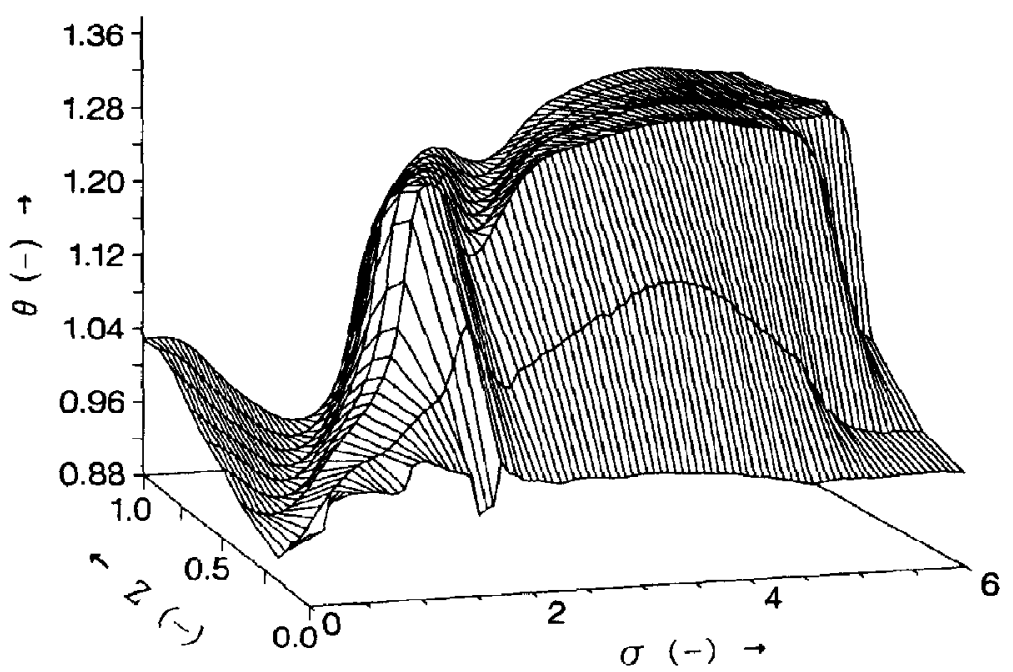

Fig. 8. Observed reactor temperature versus reactor location and time.

performed on the start-up behavior of this reactor (Verwijs et al., 1992). The calculated concentration profile of reactant $B$ as a function of time $\sigma$ over the entire reactor length $z$ is shown in Fig. 9. In this figure the lines parallel to the $\sigma$-axis represent the calculated dimensionless concentration $\Gamma_{B}$ at the dimensionless location $z$, and the lines parallel to the $z$-axis connect the data at the same moment. The $\sigma$-axis is drawn from the right side to the left side, and the $z$-axis is drawn in opposite direction, which contradicts with the temperature profile in Fig. 8 . Note the breakthrough of component $B$ around time $\sigma=2$ in Fig. 9 ( = runaway from expected reactor effluent composition!).

If the restart of the reactant $B$ feed-pump after the pump failure is considered as a second start-up, a significant difference can be observed in the reactant B concentration profiles in Fig. 9. This difference stems from the following two reasons:

- The average reactor temperature during the second start-up is much higher than for the first one.

- The reactant B feed is not ramped to its setpoint during the second start-up; see Fig. 7.

The combination of these effects results in a higher average reaction rate due to a higher initial temperature level and a higher initial concentration of reactant $B$ for the second start-up, resulting in a complete conversion of reactant $B$ at $z \approx 0.60$.

It can be concluded that for an adiabatic tubular reactor a much higher initial temperature along the reactor is required during start-up, compared to the

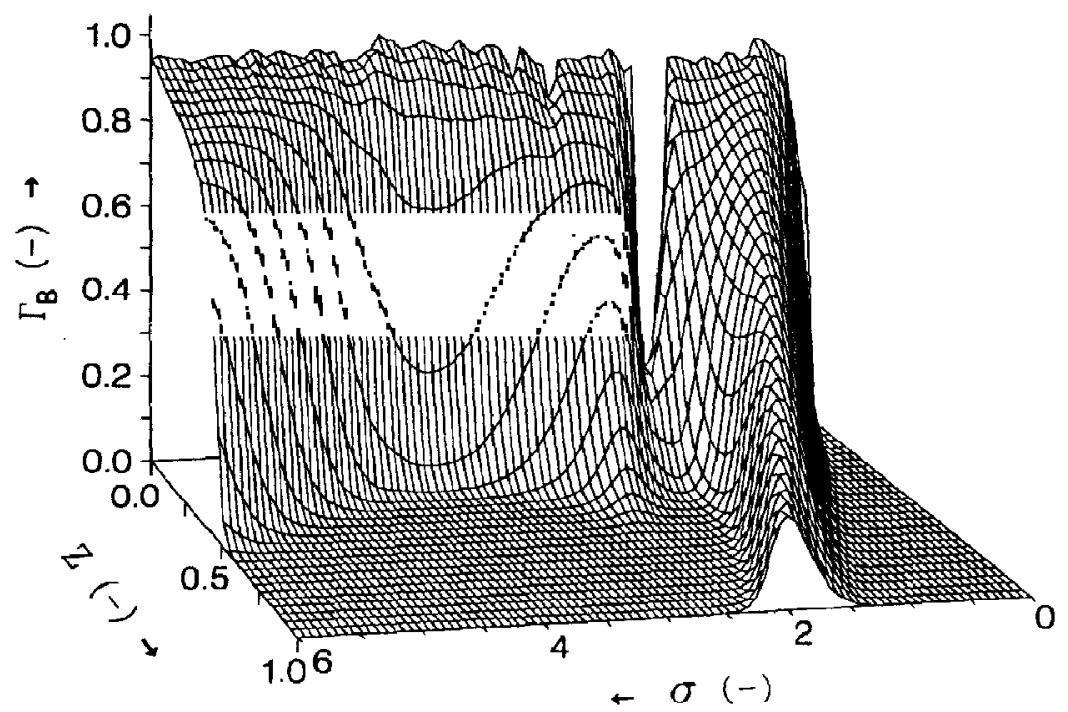

Fig. 9. Calculated reactant $B$ concentration versus reactor location and time. 
reactor inlet temperature at normal steady-state conditions, to ensure a complete reactant $B$ conversion at the reactor exit. Although, by starting up according to the policy just described, the initial temperature profile over the entire reactor is not under control!

Secondly, the spatially distributed nature of the process conditions in a tubular reactor highly complicates the reactor safeguarding during start-up operations. This can be explained by focusing again on the reactor temperature profile shown in Fig. 2. At steady-state conditions the reactor is safeguarded by monitoring the reactor inlet temperature $\left(\Delta \theta_{\text {in }}\right)$, the isothermal operation at the tail end of the reactor ( $\left.\Delta \theta_{\text {iso }}\right)$ and the adiabatic temperatute rise $\left(\Delta \theta_{\mathrm{ad}}\right)$. Additionally the reactor temperature is observed versus a maximum and minimum temperature constraint ( $\theta_{\max }$ and $\theta_{\min }$ ). The safeguarding elements $\Delta \theta_{\text {iso }}$ and $\Delta \theta_{\mathrm{ad}}$ are initially of no use when reactant $\mathrm{B}$ is introduced into the system to start up the reactor because the normal temperature profile does not exist Therefore, in industrial practice such safeguarding elements are not taken care of during the start-up, because they may generate unreasonable reactor shutdowns. As a result, all safeguarding items which may give some indication about the extent of the reaction ( $\Delta \theta_{\text {iso }}$ and $\Delta \theta_{\text {ad }}$ ) are missing and operational problems may easily arise during the start-up, as has been demonstrated in Figs $5,6,7,8$ and 9 .

Finally, the observation of a few key state variables is often sufficient for experienced operating personnel to control the process status at steady-state conditions (Verwijs et al., 1994a). A reactor is supervised in the same way by operating personnel. They use the readings of the reactant feed rates together with the values of some thermoelements to control the status of the reactor. However, the spatially distributed nature and the transient behavior of the temperature readings along the reactor make it significantly more complicated for plant operating personnel, in comparison with reactor safeguarding at steady-state conditions (a) to identify important process deviations during dynamic operations, and (b) to recognize when a process is running into hazardous situations. Again, a model-based control system, based on the reactor mass and heat balance, may provide a solution to this problem as will be shown in the next sections.

\section{START-UP STRATEGY DESIGN}

Verwijs et al. (1994a) presented a sequence of elementary intermediate operating states for the start-up of a continuously-operated adiabatic tubular reactor by taking into account the operational aspects of an entire process section. Four consecutive steps are determined to drive the reactor system from shutdown to the normal production state:

- Firstly, reactant A should be recycled over the entire process section. This process recycle loop should include the reactor feed system, the reactor, and the recovery system.
- Secondly, the reactant A recovery system should be driven to production state conditions, so the recovery system can treat the reactor effluent without significant process upsets after the start-up of the reactant $B$ feed into the reactor.

- Thirdly, the reactor temperature should be driven (simultaneously) to the required initial temperature $\theta(z, 0)$ at which reactant $B$ can be fed into the reactor safely.

- Finally, reactant B should be supplied into the reactor to start production.

The following approach is chosen to study the reactor start-up (Verwijs et al., 1994b), which is also shown schematically in Fig. 10. In the process conditioning state (step 3 of the above-mentioned sequence of operating stages) reactant $A$ is fed at a flow rate $F_{A}(0)$ into the reactor. The reactant $A$ flow is kept at this rate during the start-up of the reactant B feed pump. The reactant $B$ feed pump is initially not in service, so the reactant $B$ flow rate $F_{B}(0)=0$. The entire reactor is assumed to be at the required initial temperature level $\theta(z, 0)$. The temperature profile $\theta(z, 0)$ is assumed to be uniform over the entire reactor length $z$.

At time $\sigma=\sigma_{0}$, the reactant B feed pump is put into service, and the reactant $B$ flow is increased from zero to $F_{B}(1)$ at a constant rate in a period $\Delta \sigma_{1}$. The flow rate $F_{B}(1)$ is chosen according to the required $F_{A} / F_{B}$ flow-ratio, to control the reactant $B$ concentration at the reactor entrance to satisfy the product mix specifications.

The reactor inlet temperature $\nu_{\theta}$ has to be decreased from the initial value $\theta(z, 0)$ to the normal reactor inlet temperature $\theta(0, \sigma)$, starting at time $\sigma_{0}+\Delta \sigma_{2}$ at a rate $\alpha_{1}$. The entire process section is driven to the required production capacity target at time $\sigma_{0}+\Delta \sigma_{3}$ by increasing the reactant $B$ flow rate from $F_{B}(1)$ to $F_{B}(2)$ at a rate $\alpha_{2}$. The flow-ratio $\xi$ will be kept constant during this capacity rate increase.

In Section 5 it is demonstrated qualitatively that for an adiabatic tubular reactor a much higher initial reactor temperature is required during the start-up, compared to the reactor inlet temperature at normal steady-state conditions, to ensure a complete reactant $B$ conversion at the reactor exit during the start-up (see Figs 5-9). Therefore, questions to be addressed are:

- What is the appropriate initial temperature $\theta(z, 0)$ at which reactant $B$ can be introduced into the reactor?

- According to which trajectory should the reactor inlet temperature $\nu_{\theta}$ be decreased from the overheated initial temperature $\theta(z, 0)$ to the normal reactor inlet temperature $\theta(0, \sigma)$ ?

- At which total flow rate $\phi_{v}$ can the reactor be started up safely?

- How fast can reactant $B$ be introduced into the reactor? 


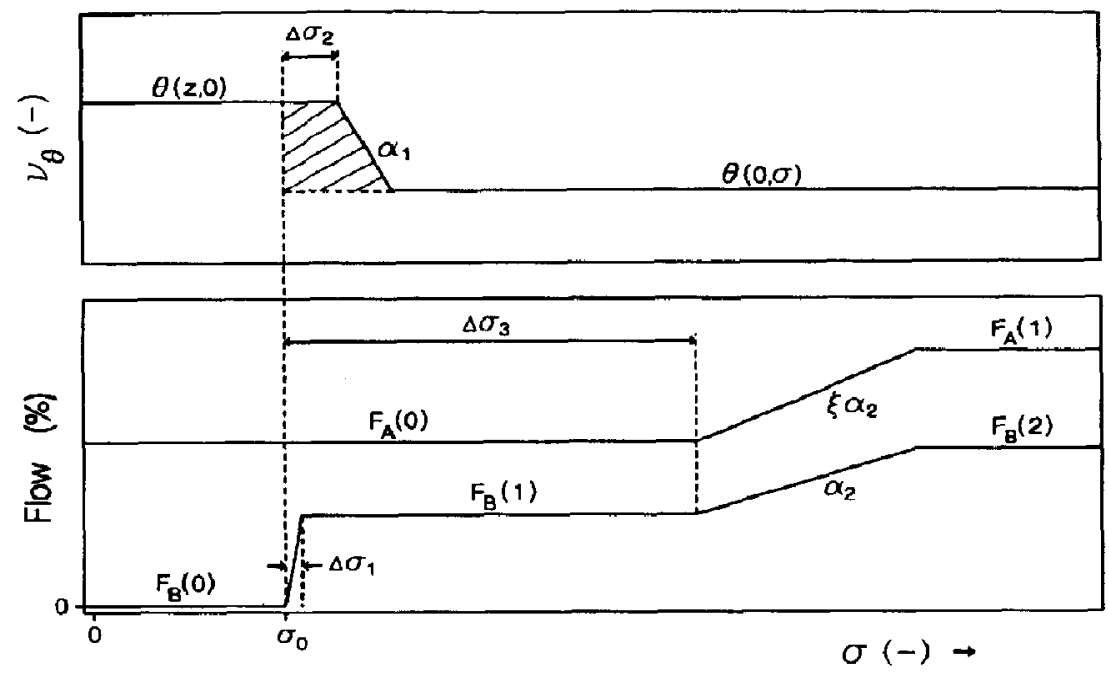

Fig. 10. Schematic representation of the manipulated variable trajectories during the reactor start-up.

A dynamic model of this industrial adiabatic tubular reactor is presented by Verwijs et al. $(1992,1994 b)$ in which the total reaction scheme has been simplified to one reaction, describing the consumption of reactant B. This reaction is assumed to be irreversible and of first order with an Arrhenius-type rate constant. The deviation of plug flow in the reactor had been described by axial dispersion, while all physical properties of the fluid were assumed to be constant over the entire reactor length. The energy take-up in the reactor vessel is included in the model, but the heat take-up in the insulation blanket and heat losses to the surroundings has been neglected.

This model is used to calculate the optimal trajectories of the manipulated variables by taking into account the operation objective of minimizing the total amount of reactant $B$ fed to the recovery section during the reactor start-up. Constraints taken into account simultaneously are: (a) the capacity of the reactor feed preheater is limited; (b) the total flow rate is constrained by the range of the flow control valve in the reactor effluent line; and (c) the maximum operating temperature of the reactor should not exceed the maximum allowable reactor temperature value $\theta_{\max }=1.35$. Some modeling results for a startup at a rate of $50 \%$ of the total plant capacity are shown in Figs 11 and 12 [see Verwijs et al. (1994b) for further details].

Two conclusions can be drawn from the dynamic modeling and optimization study:

- To maximize the reaction rate at initial start-up conditions reactant $B$ is introduced into the reactor as fast as possible and the initial reactor temperature is driven to its upper extreme simultaneously.

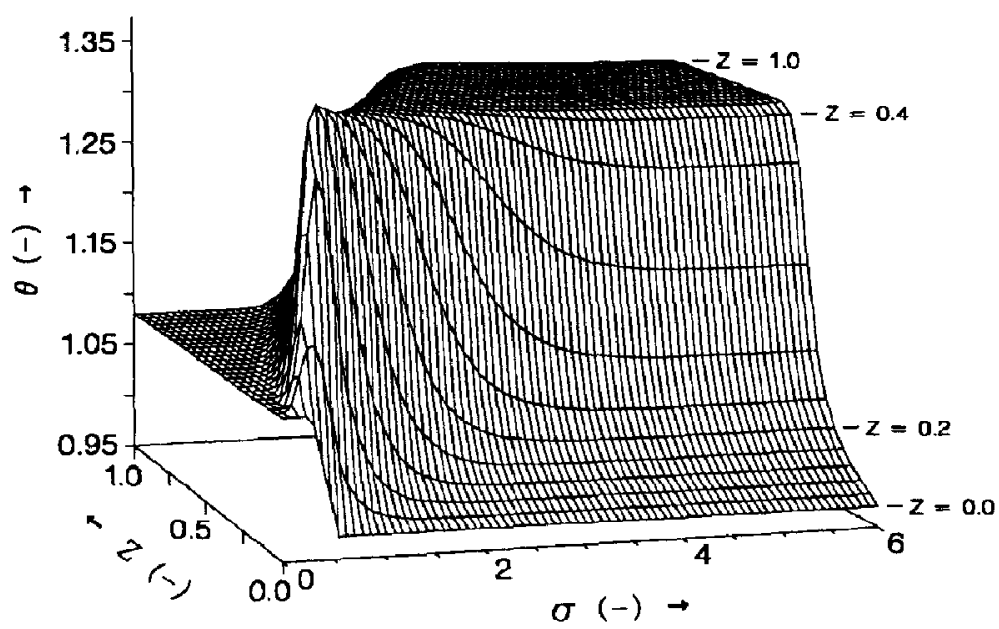

Fig. 11. Calculated reactor temperature versus reactor location and time. 


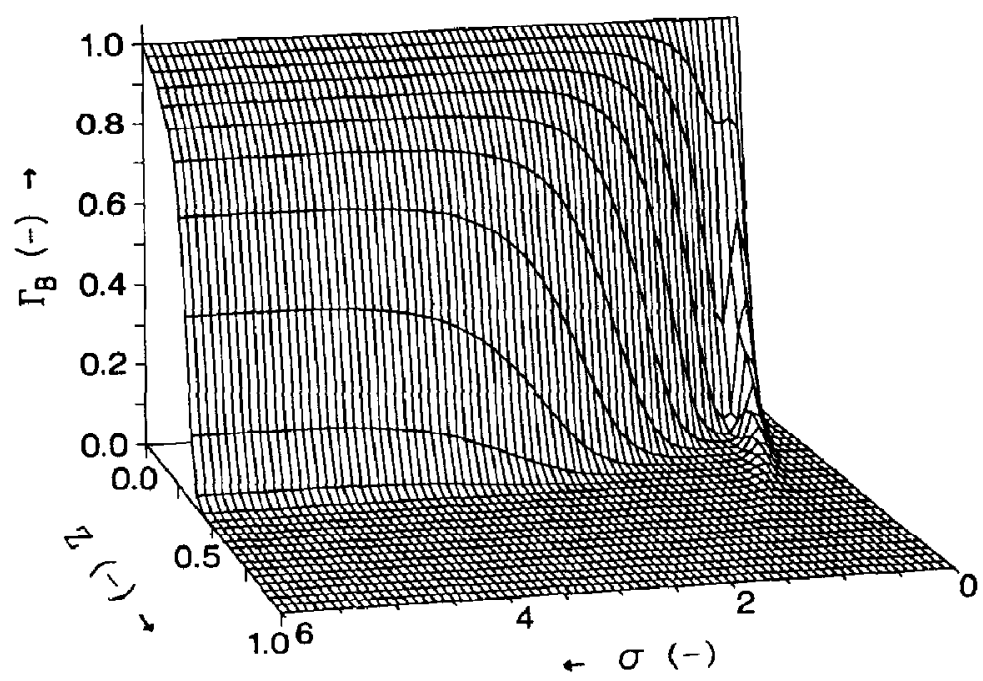

Fig. 12. Calculated reactant $B$ concentration versus reactor location and time.

- To compensate for the reaction rate decline by (a) the energy take-up in the reactor vessel and (b) the elongation of the reactant $B$ concentration profile and the reactor temperature profile due to mass and heat dispersion effects, the reactor should be overheated at initial start-up conditions. This additional heat input is determined by the shaded area in Fig. 10. This excess heat input into the reactor is maximized by taking into account the maximum allowable reactor temperature constraint.

A parameter sensitivity analysis showed that the objective of minimizing the total amount of reactant $B$ in the reactor effluent is controlled mainly by the initial reactor temperature. This is an important result because now it is possible to design a feasible reactor start-up strategy. As stated before, in the process conditioning step the reactor is driven to the initial reactor temperature $\theta(z, 0)$. This operation as such is independent of the introduction of reactant $B$ into the reactor and can be carried out very easily in a properly designed process (Verwijs et al., 1994a).

\section{IMPROVED START-UP SAFEGUARDING STRATEGY}

Conventionally, the reactor is safeguarded by supervising the instantaneous values of the process state variables within a certain operating window. It is shown in Section 5.1 that this methodology is not sufficient for chemical reactor systems because all items which may give some information about the extent of the reaction are missing during transient operations. Another shortcoming is that this methodology is not suitable for identification of imminent hazardous process conditions during process transients. Therefore an improved safeguarding methodology should provide a procedure (a) to control the manipulated variables and (b) to supervise the resulting dynamic reactor behavior.
This functionality can be introduced by controlling the actual manipulated variable trajectories versus the optimal trajectories calculated by the model. Subsequently, maximum allowable deviations from the optimal trajectories have to be determined to compensate for process disturbances and model mismatch. In an analogous way, the actual responses of the thermoelements located along the reactor can be monitored versus the optimal trajectories calculated by the reactor model, and constraints can also be defined to compensate for process disturbances and model mismatch. Now the operating personnel have a method to supervise the dynamic reactor behavior versus a target response. Consequently the reactor should be brought into a shut-down step when these constraints are exceeded to prevent the process from running into a hazardous situation.

The temperature trajectory constraints are represented by the dashed lines in Fig. 13 at several positions $z$ along the reactor. These results are obtained by varying the initial reactor temperature $\theta(z, 0)$ starting from the optimal conditions at $O(z, 0)-1.08$. The upper constraints are determined by the maximum allowable reactor temperature. The lower temperature constraint is determined by the maximum allowable reactant $B$ breakthrough. So, the temperature range available between these constraints is available for a safe reactor start-up.

The methodology described above can also be used during the design of a reactor system. Now the maximum allowable reactor temperature $\theta_{\max }$ and the maximum initial reactor temperature $\theta(z, 0)$ can be used as design parameters to enlarge the range between the upper and lower temperature constraints. Enlarging this temperature range will decrease the impact of process disturbances and model mismatch on process safety during the reactor start-up. 

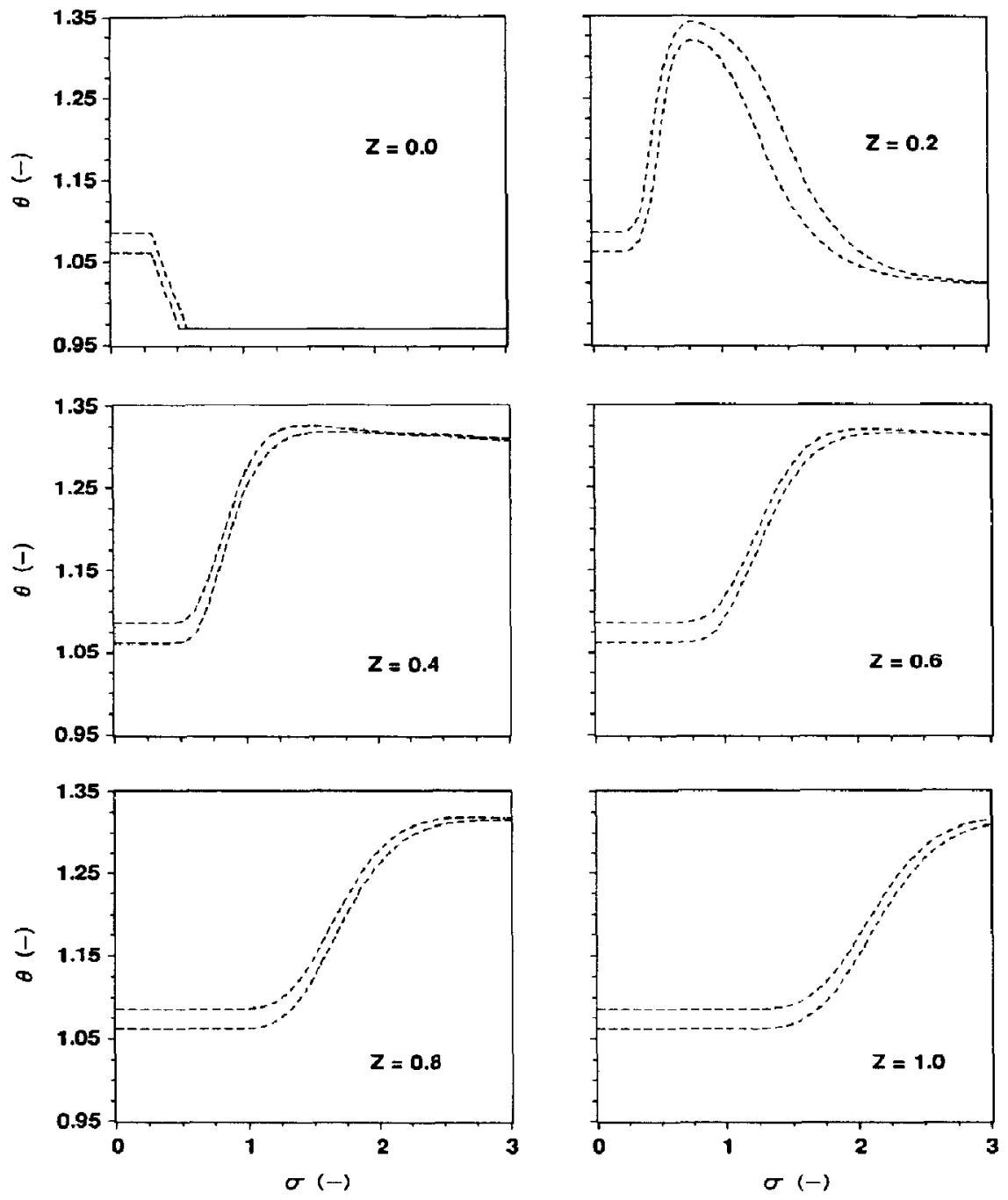

Fig. 13. Reactor temperature trajectory constraints for thermoelements located at several positions along the reactor length $z$.

In practice, the period of time up to the first maximum in the temperature trajectories is of major interest for safeguarding of the reactor start-up. After this period, the conventional safeguarding system can take over when no online model-based control and safeguarding system is available. For this particular period of time the expected thermoelement responses and their related constraints can be characterized very well by the equations which describe the response of a second-order system, with delay and complex conjugate roots of the characteristic equation, to a step change at the inlet of the system (Kuo, 1991).

\section{CONCLUSIONS}

The process safeguarding methodology used in the current industrial practice is based on controlling the instantaneous values of the process state variables within certain operating windows. If the safety con- straints are exceeded the process is brought into shutdown. Usually, these operations are executed automatically by hard-wired emergency shutdown systems. It is demonstrated, by using information of thermal runaways which occurred in industrial continuously-operated tubular reactors, that such a safeguarding system does not necessarily prevent a reactor from a runaway because excessive amounts of unreacted chemicals can still accumulate in the reactor system. A model-based control and safeguarding system is required to control the reactor mass balance in conjunction with the reactor heat balance. In this way online information can be obtained from the model about the extent of the reaction, even at abnormal process conditions.

A dynamic model of an industrial adiabatic tubular reactor is used to develop an improved safeguarding strategy for start-up operations. Two safety constraints are taken into account: (a) one of the main 
reactants has to be totally converted at the reactor exit to avoid hazardous situations in a downstream process section; and (b) the reactor temperature should never exceed a maximum allowable reactor temperature constraint.

It is concluded for the operating conditions studied that a proper control of the initial reactor temperature is critical for a safe reactor start-up. Hence, a process conditioning state for the reactor system should be included in the start-up procedure, and should be made feasible by a proper plant design.

The optimum initial reactor temperature conditions and manipulated variable trajectories are used to determine a range of operating conditions in which the reactor can be started up safely. The temperature trajectorics measured by the particular thermoelements located along the reactor can be fairly well described by the response of a second order system to a step response. Such equations can be implemented relatively easily in modern process control systems for process safeguarding purposes. Additionally, operating personnel can use such target trajectories to identify imminent hazardous situations during start-up operations.

\section{NOTATION}

\section{$C_{B} \quad$ concentration of species $B, \mathrm{~mol} / \mathrm{m}^{3}$}

$C_{B, r}$ reference concentration of species $B, \mathrm{~mol} / \mathrm{m}^{3}$

$I$ reactor length, $\mathrm{m}$

$\boldsymbol{P} \quad\left\{\boldsymbol{P} / \boldsymbol{P}_{r}\right\}$; system pressure expressed as percentage of device range, \%

$t$ time, $s$

$T \quad$ fluid temperature, $\mathrm{K}$

$T_{0} \quad$ fluid temperature at reactor inlet, $\mathrm{K}$

$T_{r} \quad$ reference temperature, $\mathrm{K}$

$v_{r} \quad$ superficial fluid velocity at reference conditions, $\mathrm{m} / \mathrm{s}$

$x$ length coordinate in direction of flow, $\mathrm{m}$

$z \quad\{x / L\}$; dimensionless reactor length

\section{Greek letters}

$\alpha_{1} \quad$ reactor inlet temperature decrease rate parameter

$\alpha_{2} \quad$ flow rate increase parameter

$\Gamma_{B} \quad\left\{C_{B} / C_{B, r}\right\}$; dimensionless concentration reactant $B$

$\xi \quad$ flow-ratio of reactants $A$ and $B$ in the reactor feed

$\theta \quad\left\{T / T_{r}\right\}$; dimensionless fluid temperature

$\theta_{\max }$ maximum allowable reactor temperature

$\theta_{\min }$ minimum allowable reactor temperature

$\Delta \theta_{\text {ad }}$ dimensionless adiabatic temperature rise

$\Delta \theta_{\text {in }}$ maximum allowable deviation of reactor inlet temperature setpoint

$\Delta \theta_{\text {iso }}$ maximum allowable temperature difference over the reactor tail-end section at normal operating conditions

$\left\{T_{0} / T_{r}\right\}$; dimensionless reactor inlet temperature $\sigma_{0}$

$\left\{\sigma_{0}=0.1\right\}$; point in time at which reactant $B$ is introduced into the reactor

$\Delta \sigma_{1} \quad$ reactant $B$ start-up rate parameter

$\Delta \sigma_{2}$ delay period before the reactor inlet temperature is ramped down

$\Delta \sigma_{3}$ delay period before the flow rate is increased to the required plant capacity target

\section{REFERENCES}

Barton, J. A. and Rogers, R. L., (Editors), 1993, Chemical Reaction Hazards - A Guide. I.Chem.E., Rugby, UK.

Bauman, E., Varma, A., Lorusso, J., Dente, M. and Morbidelli, M., 1990, Parametric sensitivity in tubular reactors with co-current external cooling. Chem. Engng Sci. 45, 1301-1307.

Eigenberger, G. and Wegerle, U., 1982, Runaway in an industrial hydrogenation reactor. ACS Symp. Ser. no. $196,133-143$.

Englund, S. M. and Grinwis, D. J., 1992, Provide the right redundancy for control systems. Chem. Engng Prog. $88(10), 36-44$.

Garrison, W. G., 1988, Major fires and explosions analyzed for 30-year period. Hydrocarb. Proc. 67(9), 115-118.

Hancock, B., 1992, Learning from accident experience - are the lessons really being applied? Loss Prev. Bull. 105, 1722.

Hosten, L. H. and Froment, G. F., 1986, Parametric sensitivity in co-currently cooled tubular reactors. Chem. Engng Sci. 41, 1073-1080.

Kuo, B. C., 1991, Automatic Control Systems, 6th Edition. Prentice-Hall International, New Jersey.

Lees, F. P., 1991, Loss Prevention in the Process Industries, Vol. 1. Butterworth-Heinemann, Oxford.

Mahoney, D. G. (Editor), 1990, Large Property Damage Losses in the Hydrocarbon-Chemical Industries. A ThirtyYear Review, 13th Edition. M\&M Protection Consultants, New York, NY. This revicw is also published in Loss Prev. Bull. 99, 1-30, (1991).

Mahoney, D. (Editor), 1993, Large Property Damage Losses in the Hydrocarbon-Chemical Industries. A Thirty-Year Review, 15th Edition. M\&M Protection Consultants, New York, NY.

Morbidelli, M. and Varma, A., 1989, A generalized criterion for parametric sensitivity: application to a pseudohomogeneous tubular reactor with consecutive or parallel reactions. Chem. Engng Sci. 44, 1675-1696.

Naess, L., Mjaavatten, A. and Li, J.-O., 1993, Using dynamic process simulation from conception to normal operation of process plants. Comput. Chem. Engng 17, $585-600$.

Raghaven, K. V., 1992, Temperature runaway in fixed-bed rcactors: online and offline checks for intrinsic safety. $J$. Loss Prev. Proc. Ind. 5, 153-159.

Shewbart, W. E., 1993, The Dow Chemical Company, July 29 , private communication on reactive chemicals incidents.

Verwijs, J. W., van den Berg, $H$. and Westerterp, $K$. R. 1992. Start-up of an industrial adiabatic tubular reactor. AIChE J. 38, 1871-1880.

Verwijs, J. W., Kösters, P. H., van den Berg, $H$. and Westerterp, K. R., 1994a, Reactor operating procedures for starl-up of continuously-operated chemical plants. $A I C h E J$. (accepted).

Verwijs, J. W., van den Berg, H. and Westerterp, K. R., 1994b, Start-up strategy design and safeguarding of industrial adiabatic tubular reactor systems. $A / C h E J$. (submitted).

Westerink, E. J. and Westerterp, K. R., 1988, Safe design of cooled tubular reactors for exothermic, multiple reactions. Multiple reaction networks. Chem. Engng Sci. 43, 1051-1069. 
Westerterp, K. R. and Ptasinski, K. J., 1984, Safe design of cooled tubular reactors for exothermic, multiple reactions. Parallel reactions. 1 -Development of criteria. Chem. Engng Sci. 39, 235-244.

Westerterp, K. R. and Overtoom, R. R. M., 1985, Safe design of cooled tubular reactors for exothermic, mul- tiple reactions. Consecutive reactions. Chem. Engng Sci. 40, 155-165.

Westerterp, K. R., Ptasinsky, K. J. and Overtoom, R. R. M., 1984, Safe design of cooled tubular reactors for exothermic, multiple first order reactions. $A C S$ Symp. Ser. no. 237, 323-335. 